\title{
Kappa opioid receptor signaling protects cartilage tissue against posttraumatic degeneration
}

\author{
Ling Wu, ${ }^{1}$ Shu Zhang, ${ }^{2}$ Ruzanna Shkhyan, ${ }^{1}$ Siyoung Lee, ${ }^{1}$ Francesca Gullo, ${ }^{1}$ Claire D. Eliasberg, ${ }^{3}$ \\ Frank A. Petrigliano, ${ }^{3}$ Kai Ba, ${ }^{2}$ Jing Wang, ${ }^{4}$ Yunfeng Lin, ${ }^{2}$ and Denis Evseenko ${ }^{1}$ \\ 'Department of Orthopaedic Surgery, Keck School of Medicine, University of Southern California, Los Angeles, California, \\ USA. '2 State Key Laboratory for Oral Diseases, West China School of Stomatology, Sichuan University, Chengdu, China. \\ ${ }^{3}$ Department of Orthopaedic Surgery, David Geffen School of Medicine, UCLA, Los Angeles, California, USA. \\ ${ }^{4}$ Department of Stomatology, Tenth People's Hospital of Tongji University, Shanghai, China.
}

\begin{abstract}
Osteoarthritis is the most common form of arthritis, and pain relief with opioid-like drugs is a commonly used therapeutic for osteoarthritic patients. Recent studies published by our group showed that the kappa opioid receptor (KOR) is highly expressed during human development in joint-forming cells. However, the precise role of this receptor in the skeletal system remains elusive. The main aim of the current study was to investigate the role of KOR signaling in synovial and cartilaginous tissues in pathological conditions. Our data demonstrate that KOR null mice exhibit accelerated cartilage degeneration after injury when compared with WT mice. Activation of KOR signaling increased the expression of anabolic enzymes and inhibited cartilage catabolism and degeneration in response to proinflammatory cytokines such as TNF- $\alpha$. In addition, selective KOR agonists increased joint lubrication via the activation of CAMP/CREB signaling in chondrocytes and synovial cells. Taken together, these results demonstrate direct effects of KOR agonists on cartilage and synovial cells and reveals a protective effect of KOR signaling against cartilage degeneration after injury. In addition to pain control, local administration of dynorphin or other KOR agonist represents an attractive therapeutic approach in patients with early stages of osteoarthritis.
\end{abstract}

Conflict of interest: The authors have declared that no conflict of interest exists.

Submitted: May 12, 2016 Accepted: November 22, 2016 Published: January 12, 2017

Reference information: JCI Insight. 2017;2(1):e88553. doi:10.1172/jci.insight.88553.

\section{Introduction}

Osteoarthritis (OA) is the most common form of arthritis, affecting approximately $15 \%$ of the population $(1,2)$. Owing to its predilection for lower extremity joints such as the knee and hip, OA is the leading cause of lower limb disability among adults, with an estimated lifetime risk of approximately $40 \%$ for men and $47 \%$ for women, and the risks are higher among individuals who are classified as obese (3). Currently, OA is one of the most commonly diagnosed diseases in general practice, with its prevalence projected to double by the year 2020 due largely to an aging population and an ever-increasing prevalence of obesity $(4,5)$.

Pain management is one of the major therapeutic approaches for OA (6). Modern opioid analgesics mainly drive their therapeutic effect through 3 classic opioid receptors: kappa opioid receptor (KOR), delta opioid receptor (DOR), and mu opioid receptor (MOR). All 3 of these receptors belong to the G proteincoupled receptor (GPCR) superfamily (7). There are 3 classes of endogenous opioid peptides, known as dynorphin, enkephalin, and endorphin, which correspond to the aforementioned receptors, respectively. All 3 peptides are capable of binding to each of the receptors with different affinities (8). Endorphin primarily binds to MOR and DOR, enkephalin to DOR, and dynorphin to KOR. Activation of opioid receptors by endogenous or exogenous agonist in neurons is associated with the modulation of adenylyl cyclase activity (9), the opening of potassium channels (10), the inhibition of calcium channels (11), and the activation of mitogen-activated protein kinases (MAPKs) (12-14).

Since KOR agonists typically do not have the same addictive properties as MOR-activating opioids, KOR signaling has drawn much more attention in the development of nonaddictive analgesics that do not activate the reward pathways stimulated by morphine-like opioids (15). However, the therapeutic potential of KOR agonists could reach far beyond their analgesic properties. It has been shown that activation of KOR signaling by a chemical agonist may have antiinflammatory effects in arthritic conditions (16), although the exact mechanisms of these effects remain unclear. Recently the expression of KOR was 
reported in cartilage (17), although the function of KOR signaling in cartilage development and cartilage matrix homeostasis is largely unknown.

In this study, we demonstrate that KOR is expressed in human cartilage cells throughout ontogenesis. We also show that KOR stimulation induces secretion of lubricin by chondrocytes and plays both protective and anabolic roles in cartilage tissue. This study demonstrates potentially novel biological functions of KOR outside of the neural system and suggests that this receptor has broader functions than analgesia, mood control, and neuroprotection.

\section{Results}

KOR and prodynorphin are both expressed in human fetal and adult synovial joints. We have previously reported a number of genes that are enriched in human cartilage-committed mesenchymal cells (prechondrocytes) by microarray analysis when comparing prechondrocytes with total limb cells (17). The gene encoding KOR, Oprk1, showed the highest enrichment relative to other genes. At 5 to 6 weeks of human development, immunohistochemical analysis of developing limbs showed that KOR was strongly expressed in the cartilage tissue in mesenchymal condensations (Figure 1A). Prodynorphin (PDYN), which is the precursor of the 2 isoforms of the endogenous ligand of KOR, dynorphin A and $\mathrm{B}$, was also expressed in cartilage at this stage. KOR and PDYN are expressed on blood vessel-like structures, which emerge on 8- to 12-weekold fetal limbs in the areas surrounding developing joints (Supplemental Figure 1A; supplemental material available online with this article; doi:10.1172/jci.insight.88553DS1). At 17 weeks of human development, synovial joints contain well-defined structures of articular cartilage and vascularized synovial membrane. At this stage, the expression of KOR expanded from cartilage to also include the synovial blood vessels, while the expression of PDYN completely shifted from cartilage to synovial blood vessels (Figure 1B).

During adulthood, both KOR and PDYN were expressed in the blood vessels in synovial membranes (Figure 1C). More specifically, KOR was found in both veins and arteries, while PDYN was mainly expressed by perivascular cells in arterial walls (Supplemental Figure 1B). Microvessels were highly enriched on the surface of synovial membrane facing the joint cavity (Supplemental Figure 1C). To determine which population expresses PDYN, fluorescence-activated cell sorting (FACS) was performed to sort out 4 mesenchymal populations from adult human synovial membrane (Supplemental Figure 1D): CD31-

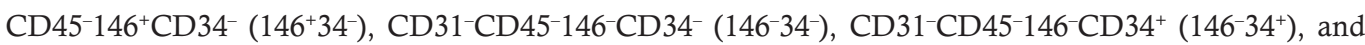
$\mathrm{CD}^{2} 1^{-} \mathrm{CD} 45^{-} 146^{+} \mathrm{CD} 34^{+}\left(146^{+} 34^{+}\right)$. Expression levels of $P D Y N$ in $146^{+} 34^{-}$cells were significantly higher than those observed in the other 3 populations (Figure 1D). This particular immunophenotype has previously been associated with vascular pericytes (18). Meanwhile, KOR-positive cells were mainly located in the superficial zone on the articular surface of adult cartilage, while PDYN was absent in both the superficial zone and the deep zone of cartilage (Figure 1E and Supplemental Figure 1E).

Oprk1 $1^{-1-}$ mice have more severe matrix degradation than WT after knee injury. To further characterize the function of KOR/dynorphin signaling, we utilized knockout mice for both KOR and dynorphin. Neither KOR knockout mice nor dynorphin knockout mice showed major deformity in limb development (19, 20). Therefore, we hypothesized that KOR signaling might play an important role in pathological conditions. To mimic acute injury in knee joints, we performed an open knee surgery on WT and Oprk1 ${ }^{-/-}$mice to create full-thickness cartilage and subchondral bone defects in the trochlear groove as described previously (20). One week after injury, both mice showed severe cartilage degradation surrounding the defect site (Figure 2A). Notably, the expression of KOR in WT increased at 1 week after surgery, indicating that KOR signaling is endogenously elevated after injury. At 4 weeks after surgery, safranin $\mathrm{O}$ staining indicated that injury induced obvious glycosaminoglycan (GAG) loss on the femoral condyle of Oprk1 $1^{-/-}$mice, while GAG content did not change in WT mice (Figure 2B). Defects in the trochlear groove left cracks on the articular surface at week 4 and cartilage tissue surrounding the defects was completely degraded at week 8 after surgery (Supplemental Figure 2). Osteoarthritis Research Society International (OARSI) scores (21) on medial femoral condyles were significantly higher in Oprk1 ${ }^{-1-}$ mice than in WT (Figure 2C), indicating a much more severe form of arthritis in genetically modified animals. Numbers of chondrocytes in the medial femoral condyle also significantly decreased by week 4 after injury (Figure 2D). To better understand the endogenous response of the KOR/dynorphin signaling system to injury, expression of PDYN was examined in synovial membrane 1 week after joint surface injury. IHC demonstrated that the expression levels of PDYN in both WT and KO mice were substantially upregulated after injury (Supplemental Figure 3). 
A 5-6 week
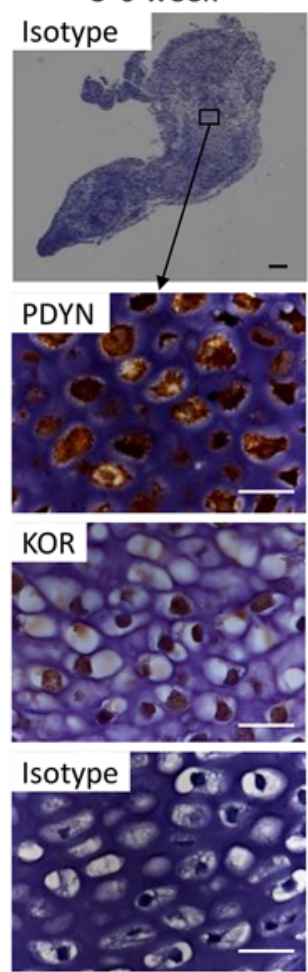

D

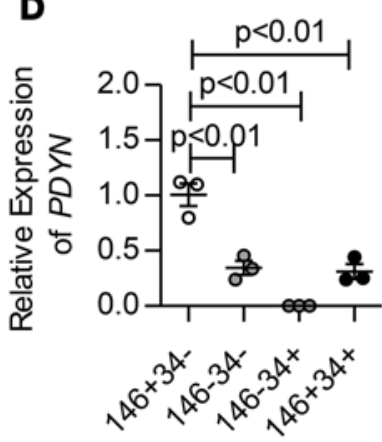

B

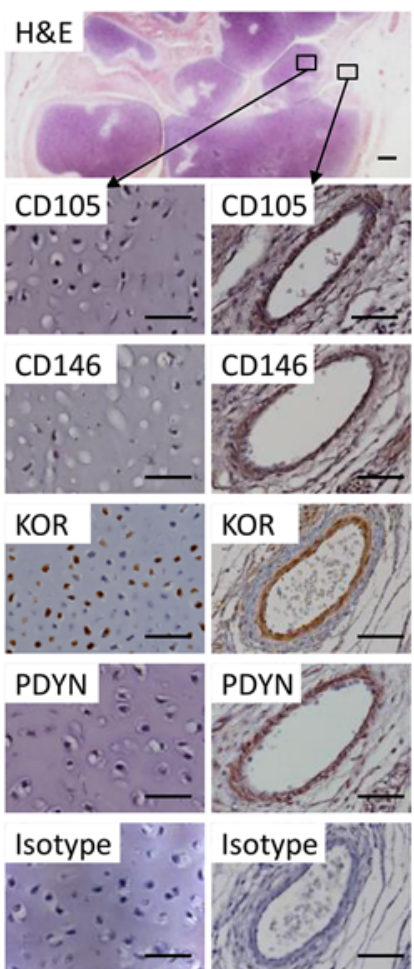

E KOR
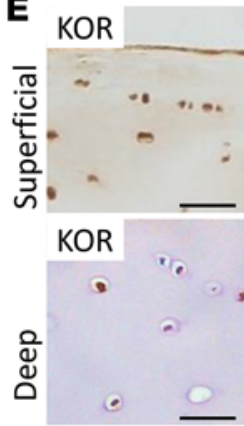

C adult

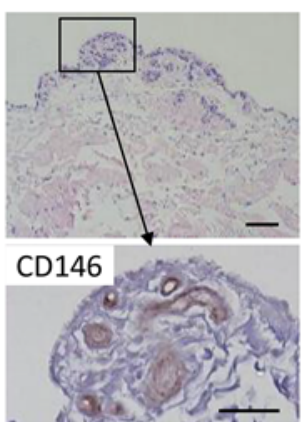

PDYN

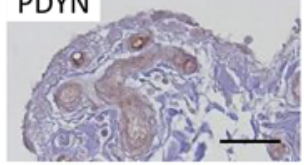

KOR
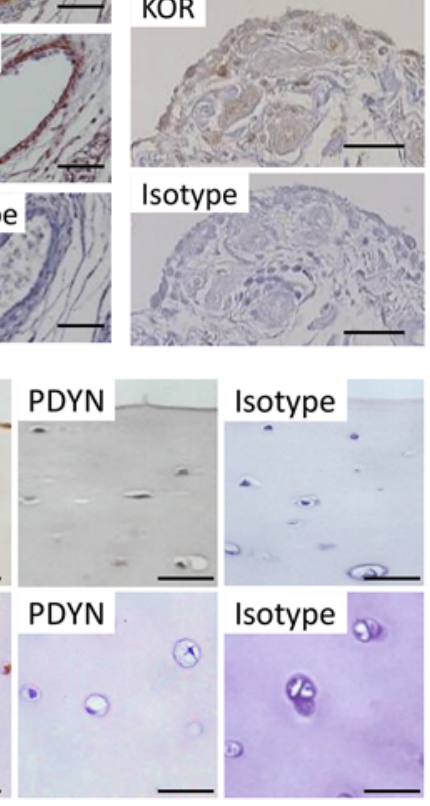

Figure 1. KOR and PDYN are expressed in synovial and cartilage tissue during development of human limbs. (A) Immunohistochemistry was performed on 5- to 6-week-old human fetal upper limbs for detection of prodynorphin (PDYN) and kappa opioid receptor (KOR). Scale bars: $20 \mu \mathrm{m}$. (B) Expression of CD105, CD146, PDYN, and KOR in 17-week-old human palm. Scale bars: $20 \mu \mathrm{m}$. (C) Expression of CD146, PDYN, and KOR in human adult synovial membrane. Scale bars: $50 \mu \mathrm{m}$. (D) Real-time PCR confirmed the expression of PDYN in pericytes within the synovial tissue. Mean $\pm \operatorname{SD}(n=3)$. Each data point represents the average of 3 technical repeats from 1 tissue donor. $P$ values were calculated with 1-way ANOVA followed by Tukey honest significant difference post-hoc test. $146^{+} 34^{-}, \mathrm{CD}^{-} 1^{-} \mathrm{CD} 45^{-} 146^{+} \mathrm{CD}^{-} 4^{-}$;

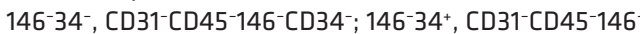

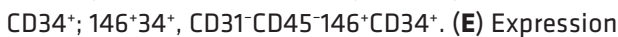
pattern of KOR protein is different between the superficial zone $(0-50 \mu \mathrm{m})$ and the deep zone $(50-100 \mu \mathrm{m})$ of human adult cartilage tissue. Scale bars: $20 \mu \mathrm{m}$. Images from all panels except $\mathbf{D}$ show representative data from 1 of 3 independent experiments ( 3 different donors).

Oprk1 $1^{-1-}$ mice possess higher levels of proinflammatory cytokines and catabolic enzymes in injured joints. To better determine the mechanism by which KOR signaling protects cartilage from degradation after injury, we evaluated levels of known inflammatory cytokines and catabolic enzymes by IHC. Staining was performed for TNF- $\alpha$, IL-1 $\beta$, MMP3, MMP13, and ADAMTS4 and -5 on sagittal sections of WT and $O p r k 1^{-1-}$ mice knee joints 1 week after injury. Our data indicate that Oprk1 $1^{-1-}$ mice expressed significantly higher levels of inflammatory cytokines in addition to increased levels of catabolic enzymes relative to WT animals (Figure 3 ).

This finding prompted us to further investigate whether activation of KOR could inhibit expression of the catabolic enzymes after the exposure to procatabolic factors. Human cartilage explants were cultured in $10 \mathrm{ng} / \mathrm{ml} \mathrm{TNF-} \alpha$ to mimic cartilage degradation in vitro, as TNF- $\alpha$ is a well-accepted inducer of catabolism in cartilage (22). KOR signaling was activated by adding $1 \mu \mathrm{M}$ dynorphin $\mathrm{A}$ in the culture medium. TNF- $\alpha$ treatment markedly induced loss of GAGs, while the presence of dynorphin A substantially mitigated the destructive effects of TNF- $\alpha$ (Figure 4A). Additionally, total RNA was extracted from cartilage explants and expression levels of genes encoding catabolic enzymes were quantified by real-time PCR. Levels of several catabolic genes including $M M P 3, M M P 13$, ADAMTS4, and ADAMTS5 were significantly higher in the TNF- $\alpha$-induced group, while dynorphin A treatment inhibited the TNF- $\alpha$-induced expression of these catabolic enzymes (Figure 4B). We next measured total collagen content by hydroxyproline assay, and total GAG content was quantified by a 1,9-dimethylmethylene blue (DMMB) assay as previously described (23). Both results demonstrated that TNF- $\alpha$ degraded cartilage matrix components, while dynorphin A significantly reduced TNF- $\alpha$-induced matrix degradation (Figure 4, C and D). Finally, dynorphin A showed protection of chondrocytes from TNF- $\alpha$-induced apoptosis (Figure 4, E and F), as indicated by annexin V staining followed by flow cytometry.

KOR agonists increase lubricin expression in chondrocytes through the $A M P / C R E B$ signaling pathway. Lubricin, also known as proteoglycan 4 (Prg4) or superficial zone protein (SZP), is secreted by articular chondrocytes and synovial cells and is believed to play a role in modulating inflammatory responses in synovial joints (24, 25). Therefore, we investigated the expression of lubricin in mice 1 week after injury. Compared with WT, lubricin expression was significantly lower in $\mathrm{Oprk1}^{-{ }^{--}}$mice (Figure 5, A and B). Chondrocytes isolated from 
A

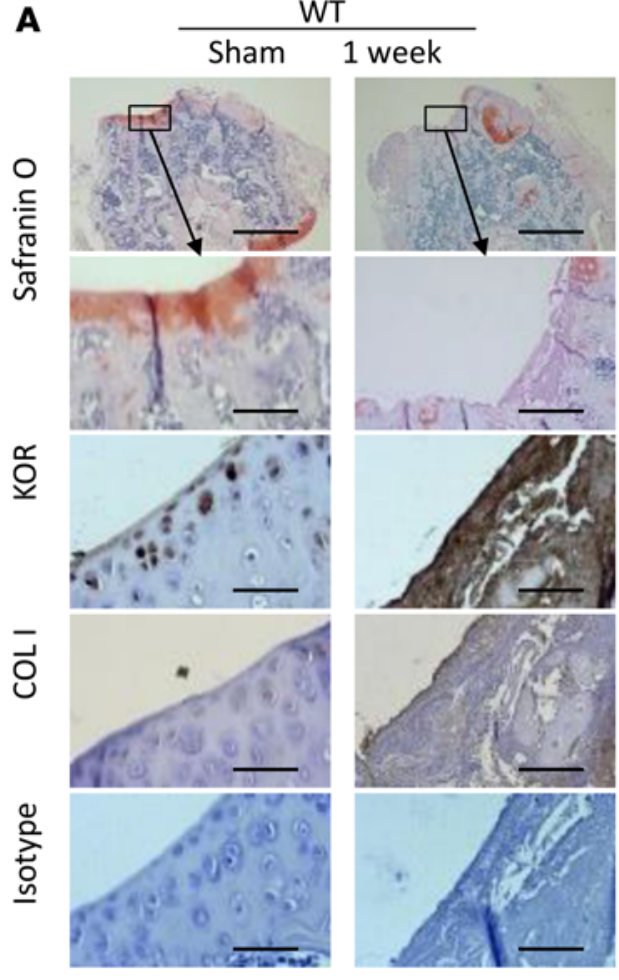

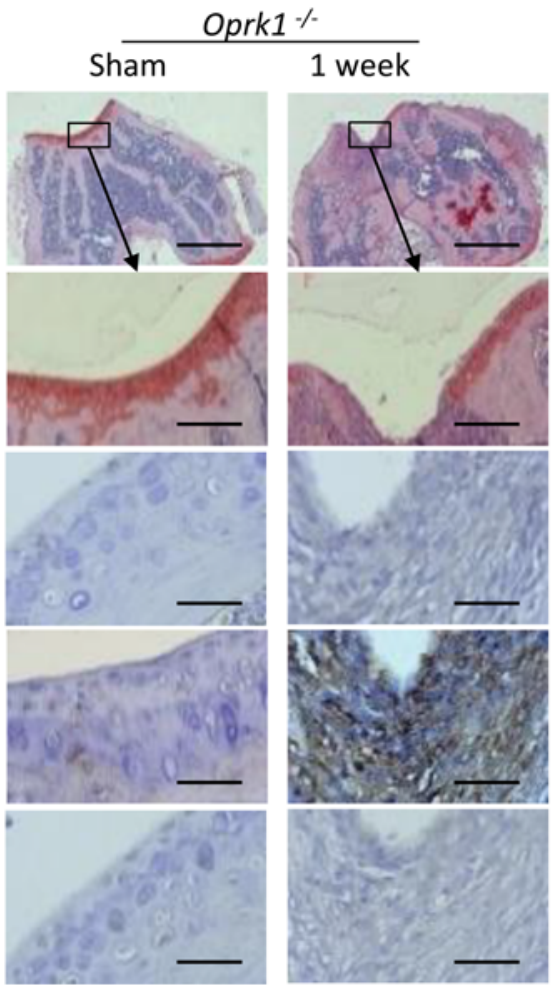

$\circ$ WT

Figure 2. Kappa opioid receptor knockout (Oprk1-/-) in mice accelerates glycosaminoglycan loss after knee joint injury. (A) Transverse plane sections of mouse knee joints at 1 week after injury. Safranin $\mathrm{O}$ staining was performed to evaluate the glycosaminoglycan (CAG) loss. Scale bars: $500 \mu \mathrm{m}$ in upper panel, $100 \mu \mathrm{m}$ in middle and lower panels. (B) Sagittal plane sections of mouse knee joints show medial femoral condyle and tibial plateau. Arrows indicate the regions of cartilage with low GAG expression. Scale bars: $100 \mu \mathrm{m}$. (C) Severity of osteoarthritis was examined using the Osteoarthritis Research Society International (OARSI) score on sagittal sections of medial femoral condyle. Mean $\pm \operatorname{SD}(n=6)$. Each data point represents the average of 3 scorings of 1 mouse.(D) Number of chondrocytes in articular cartilage was counted on sagittal sections of medial femoral condyle. Mean \pm SD $(n=6)$. Images in $\mathbf{A}$ and $\mathbf{B}$ are representative samples from a total of 6 . $P$ values in $\mathbf{C}$ and $\mathbf{D}$ were calculated using Student's $t$ test. COL I, collagen I; KOR, kappa opioid receptor.

B

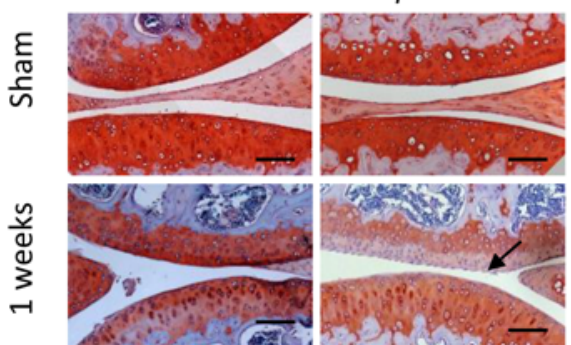

C
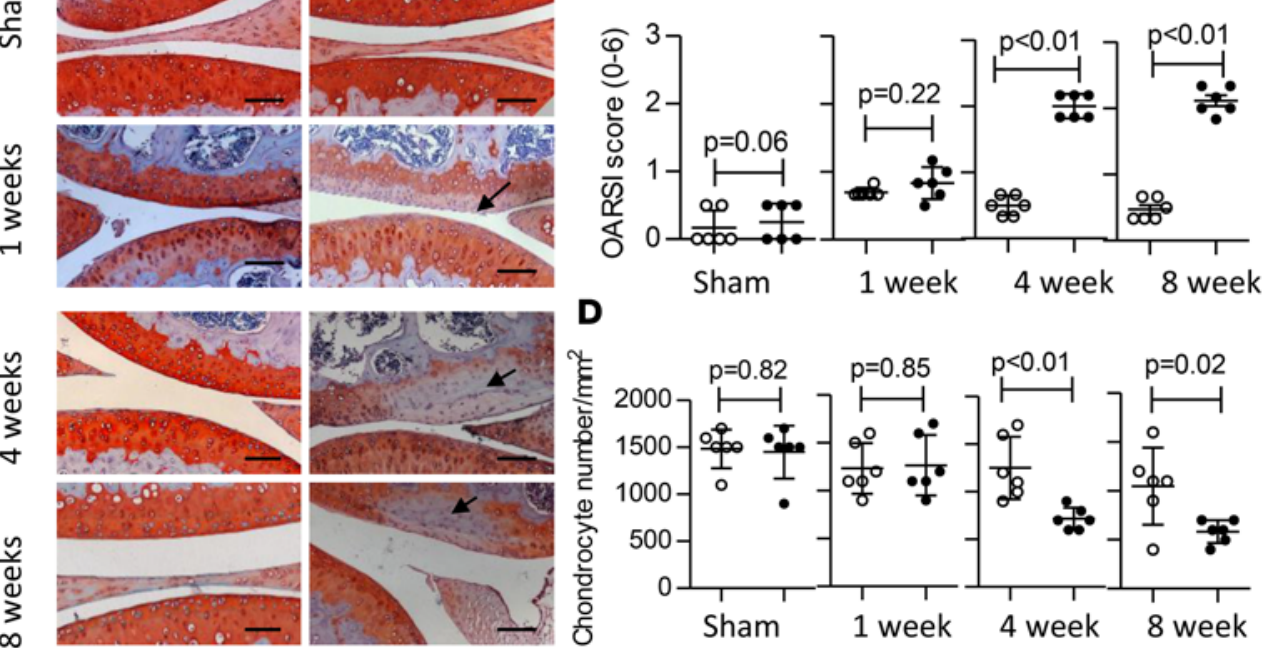

WT and Prkk $^{-/-}$mice rib cartilage were then cultured in the medium with or without $1 \mu \mathrm{M}$ dynorphin A. Western blot analysis indicated that only dynorphin A-treated WT chondrocytes produced lubricin, while Oprk1 $1^{-/}$chondrocytes failed to activate lubricin production in response to dynorphin A treatment (Figure 5C and Supplemental Figure $4 \mathrm{~A}$ ). To validate this lubricininducing activity in humans, Western blot was performed on fetal chondrocytes treated with $0,0.1$, or $1 \mu \mathrm{M}$ dynorphin A for 24 hours (Figure 5D and Supplemental Figure 4B). Quantitative PCR was also carried out on fetal chondrocytes

treated with $1 \mu \mathrm{M}$ dynorphin A for 24 hours (Figure 5E). Activation of KOR significantly increased the expression of the PRG4 gene and the protein levels of lubricin. To gain further understanding of how KOR signaling induces PRG4 expression we explored the activity of known downstream effectors of the pathway. KOR is known to activate the Ga subunit of GPCR and thereby increase the intracellular cyclic AMP (cAMP) concentration (26) and subsequently activate downstream signaling via phosphorylation of cAMP response element binding protein (CREB). Based on the previously published literature, we hypothesized that KOR regulates lubricin expression by interacting with the CAMP/CREB signaling pathway in chondrocytes. To test our hypothesis, we treated porcine articular chondrocytes with the KOR agonist U50,488H for 0, 1, 2, 24, and 48 hours (27). Phosphorylation of CREB (p-CREB) at the serine 133 residue, as detected by Western blot, increased significantly in chondrocytes after 24 hours of treatment with U50,488H (Figure $5 \mathrm{~F}$ and Supplemental Figure 4C). Our data indicate that both dynorphin A and forskolin, a classic inducer of cAMP 

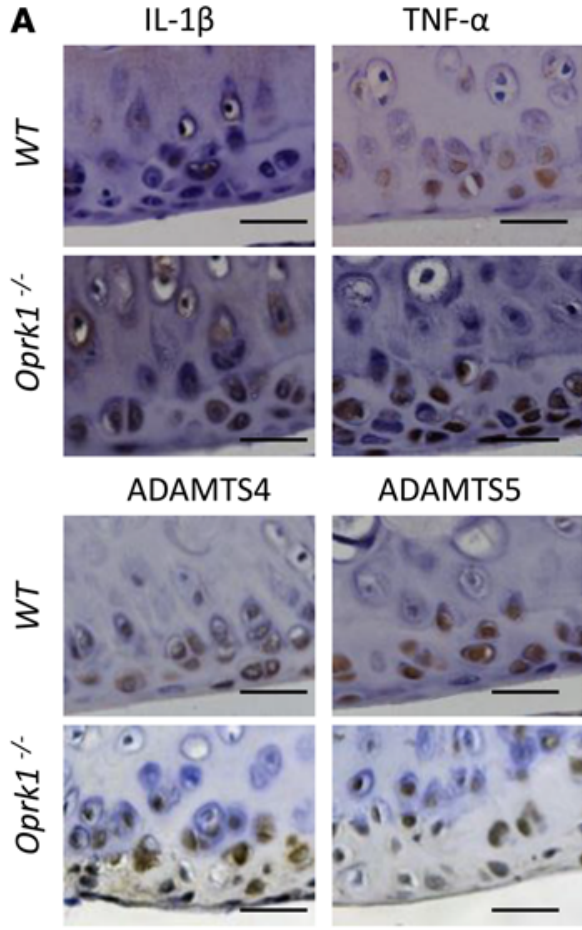

ADAMTS5
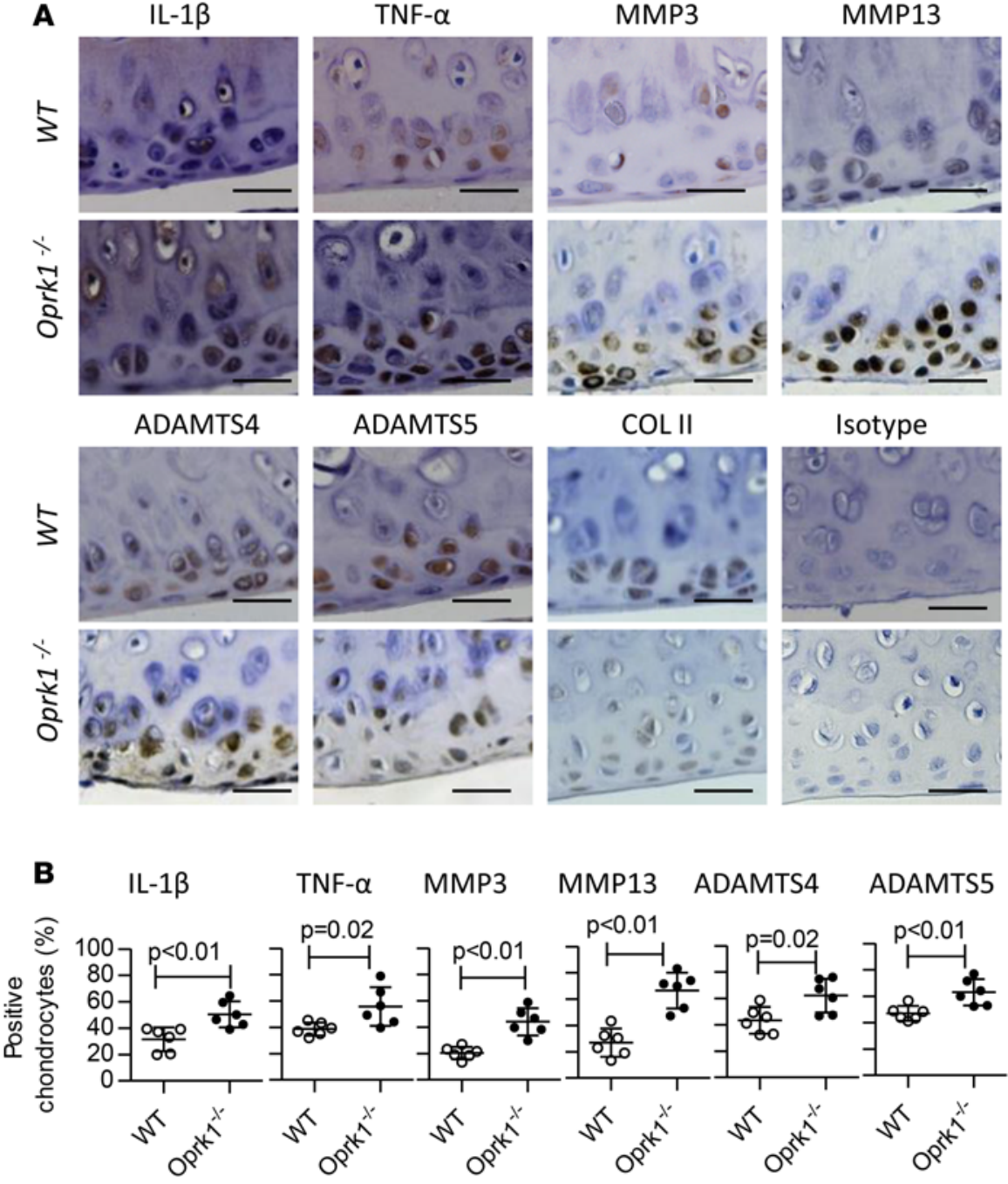

Figure 3. The expression of catabolic enzymes and proinflammatory cytokines in articular cartilage tissue of kappa opioid receptor knockout (Oprk1/-) and WT mice. (A) Immunohistochemical staining was performed on sagittal sections of medial femoral condyle. Scale bars: $20 \mu \mathrm{m}$. Representative images from 6 independently analyzed mice are shown. (B) Quantification of positively stained chondrocytes. Mean $\pm \mathrm{SD} n=6$. $P$ values were calculated using Student's $t$ test. Positive signal is shown in brown color. Each data point represents an average of 3 counts from each mouse. COL II, collagen II.

and p-CREB levels, increased the intracellular concentration of cAMP in chondrocytes (Figure 5G). Twenty-four-hour treatment with forskolin significantly increased phosphorylation of CREB, as well as the expression of lubricin in cultured chondrocytes (Figure $5 \mathrm{H}$ and Supplemental Figure 4, D and E). Indeed, inhibition of PKA activity by the chemical inhibitor KT 5720 (19) reduced phosphorylation of CREB and the expression of lubricin induced by dynorphin (Figure 5I and Supplemental Figure 4, F and G). To further validate this mechanism of action, we inhibited the activity of protein kinase A (PKA) by chemical inhibitor and small interfering RNA (siRNA). PKA is activated by cAMP and phosphorylates CREB (28). Meanwhile, we also used siRNA to knockdown catalytic subunits $\alpha$ and $\beta$ of PKA. Our data indicate that only expression of subunit $\alpha$ of PKA was significantly reduced by siRNA (Supplemental Figure 4, H and I). Knockdown of subunit $\alpha$ of PKA reduced phosphorylation of CREB and the expression of lubricin induced by dynorphin (Figure 5J and Supplemental Figure 4, F and G). Together, the results of both chemical inhibition and RNA interference suggest that lubricin production in chondrocytes induced by dynorphin A is PKA and p-CREB dependent.

Activation of KOR signaling enhances GAG synthesis in cartilage from $O A$ patients. To examine whether activation of KOR can increase GAG biosynthesis in chondrocytes, human fetal chondrocytes were cultured with or without $1 \mu \mathrm{M}$ dynorphin A for 1 week. Conditioned medium was collected and total GAG content was precipitated from the conditioned medium. Results of the DMMB assay indicate that 7-day treatment with dynorphin A doubled the amount of GAG secreted from chondrocytes (Supplemental Figure 5, A and B). Real-time PCR was next performed to examine the expression of genes encoding critical enzymes in the chondroitin sulfate biosynthetic chain (29). Xylosyltransferase I (XYLT1), $\beta$-1,4-galactosyltransferase, polypeptide 1 ( $\beta 4 G A L T 1), \beta$-1,3-galactosyltransferase polypeptide 6 ( $\beta 3 G A L T 6), \beta$-1,3-glucuronyltransferase 3 ( $\beta 3 G A T 3)$, and UDP- $N$-acetyl- $\alpha$-D-galactosamine:polypeptide $N$-acetylgalactosaminyltransferase 1 (GALNT1) were upregulated in chondrocytes treated with dynorphin A (Supplemental Figure 5, C-G). The concentration of dynorphin in synovial fluid from OA patients and normal individuals was measured by ELISA. The concentration of dynorphin (both A and B forms) in healthy individuals was significantly higher than in OA patients (Figure 6A). This result indicates that dynorphin/KOR signaling may be implicated in the pathogenesis of OA. Next, OA cartilage explants were treated with $\mathrm{U} 50,488 \mathrm{H}$ to determine if this treatment enhances GAG synthesis in the diseased cartilage. Quantification of GAGs and Alcian blue staining revealed that $\mathrm{U} 50,488 \mathrm{H}$ treatment increased the GAG content in cartilage explants (Figure $6, \mathrm{~B}$ 
A Control

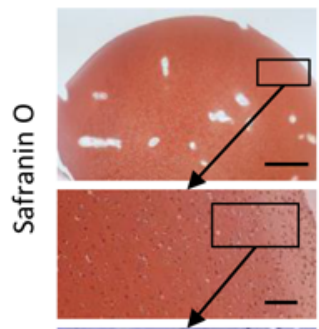

$\sum_{\Sigma}^{m}$
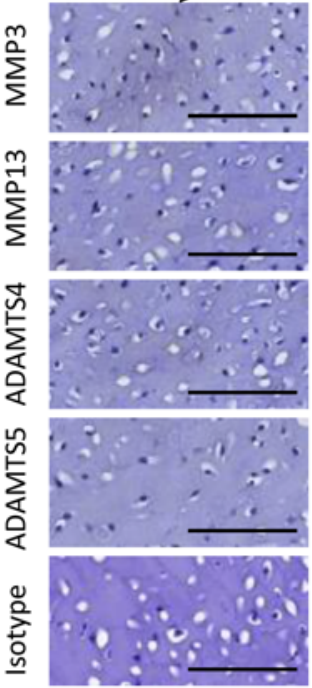

E

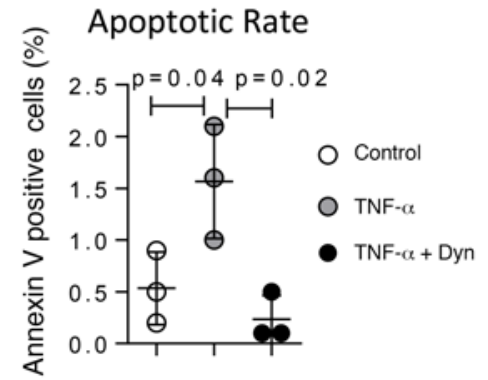

TNF- $\alpha$
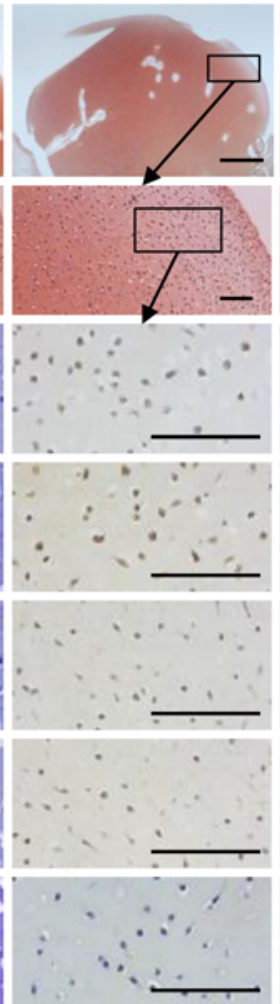

F

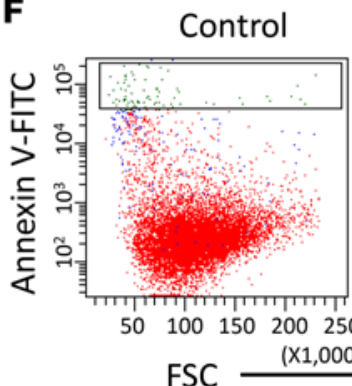

B

O control O TNF- $\alpha \quad \bullet$ TNF- $\alpha+$ Dyn

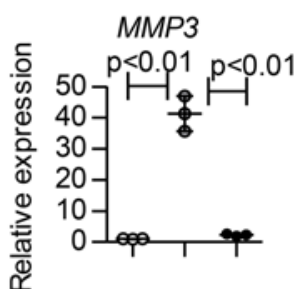

MMP13
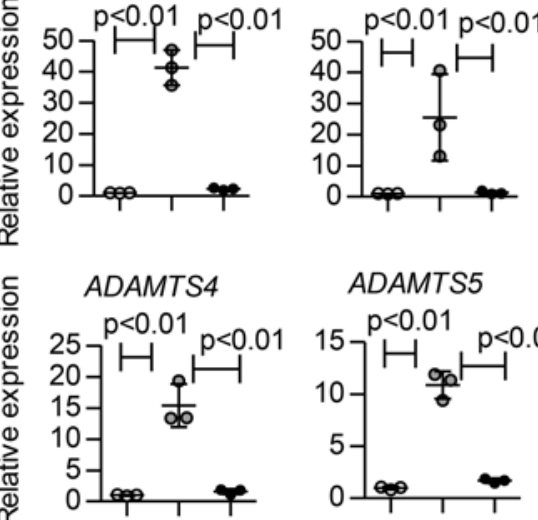

\section{ADAMTS5}

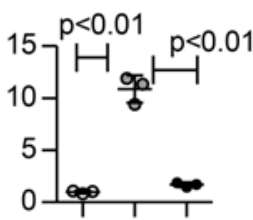

C

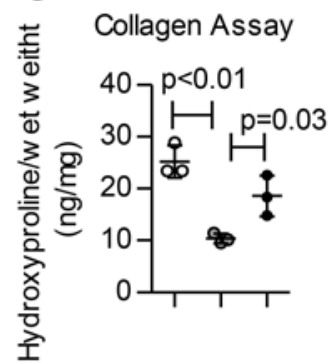

D

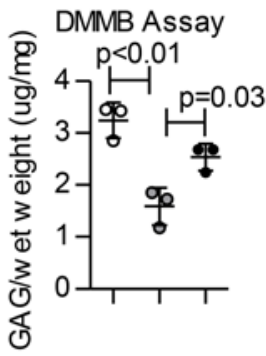

TNF- $\alpha$

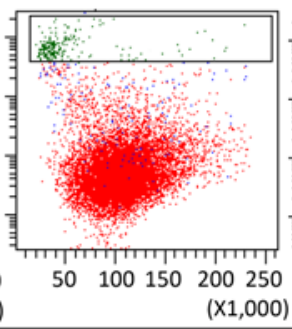

TNF- $\alpha+$ Dyn

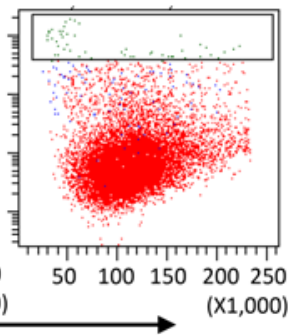

Figure 4. Dynorphin A inhibited the catabolic effects of TNF- $\alpha$ in cartilage explants. (A) Cartilage explants were treated with basal medium or basal medium supplemented with TNF- $\alpha(10 \mathrm{ng} / \mathrm{ml})$ alone or basal medium with TNF- $\alpha(10 \mathrm{ng} / \mathrm{ml})$ and dynorphin $A(1 \mu \mathrm{M})$. Dynorphin A attenuated glycosaminoglycan (GAG) loss and the activation of catabolic enzymes caused by TNF- $\alpha$. Scale bars: $500 \mu \mathrm{m}$ for top row of safranin 0-stained sections, 100 $\mu \mathrm{m}$ for all other images. (B) Real-time PCR was performed to examine the expression levels of catabolic genes in cartilage explants. Mean \pm SD $(n=3)$. (C) Hydroxyproline assay was performed to measure total collagen in cartilage explants treated with TNF- $\alpha$ or TNF- $\alpha+$ dynorphin A. Mean \pm SD ( $n=3$ ). (D) 1,9-Dimethylmethylene blue (DMMB) assay was used to determine the GAG content in cartilage explants. Mean \pm SD $(n=3)$. (E and F) Dynorphin A inhibits TNF- $\alpha$-induced apoptosis in articular chondrocytes. Mean \pm SD $(n=3)$. $P$ values in all panels were calculated with 1 -way ANOVA followed by Tukey honest significant difference post-hoc test. Each data point represents an average of 3 counts from each tissue donor. Images shown in $\mathbf{A}$ and $\mathbf{F}$ are representative of 3 independent experiments (3 different tissue donors). FSC, forward scatter.

and C). Real-time PCR analysis of the same tissue demonstrated that major genes involved in the biosynthesis of chondroitin sulfate were upregulated in OA explants treated with U50,488H (Figure 6D).

\section{Discussion}

In our previous study, high expression of the Oprk1 gene encoding KOR was shown in developing human limbs (17). However, no skeletal phenotype was found in Oprk1-- mice (19). Genetic ablation of the other opioid receptors also caused no skeletal abnormalities $(30,31)$. It was proposed that the function of one opioid receptor might be compensated by other opioid receptors, owing to their structural and functional 
A

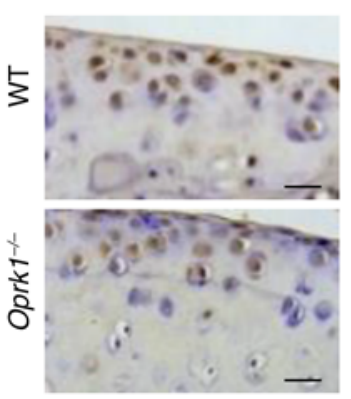

C

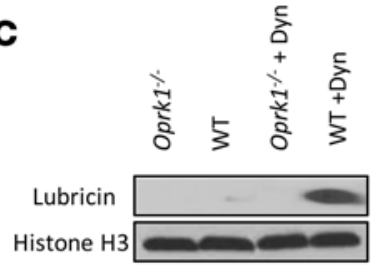

Week 1

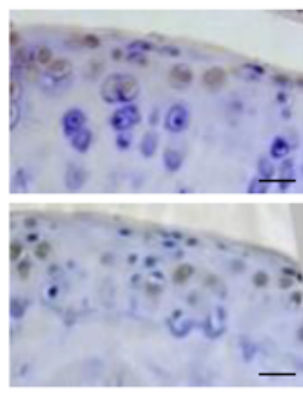

D
Week 4

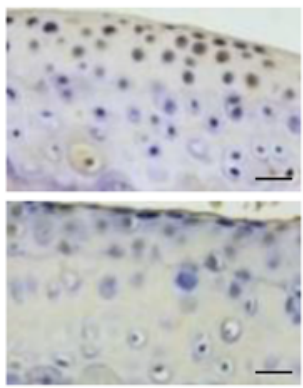

B
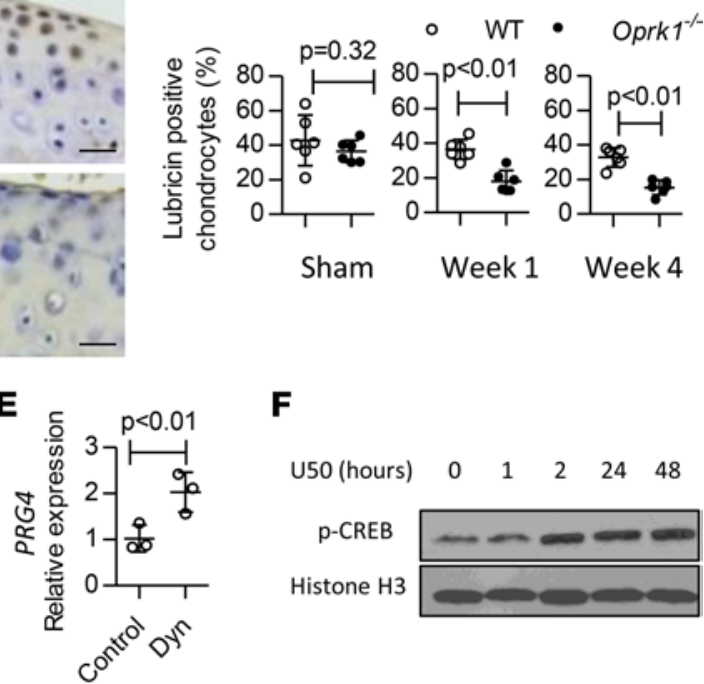

$\mathbf{F}$

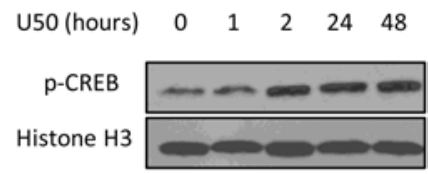
Histone $\mathrm{H} 3-2-$

H

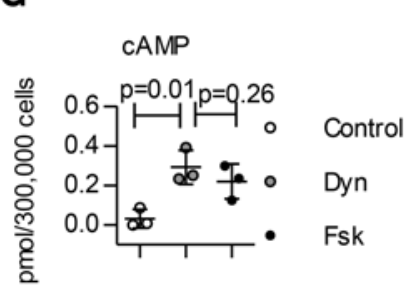

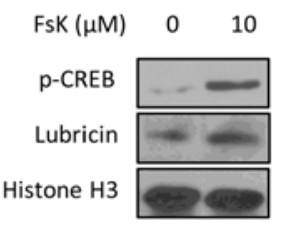

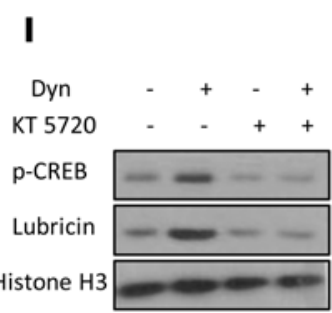

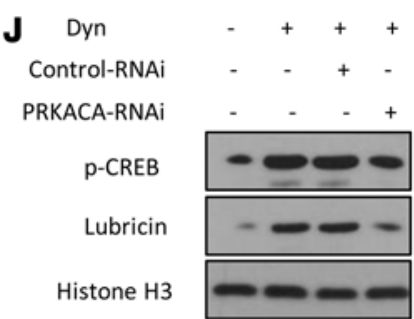

Figure 5. Dynorphin A increases the expression of lubricin/PRG4 in chondrocytes. (A) Immunohistochemistry for lubricin was performed on sagittal sections of medial femoral condyles from WT or kappa opioid receptor (KOR) 1 knockout (Oprk1 $1^{-1}$ ) mice. Scale bars: $20 \mu \mathrm{m}$. Images are representative samples from a total of 6. (B) Lubricin-positive chondrocytes were counted on sagittal sections of femoral condyle. Mean \pm SD $(n=6)$. Each data point represents the average of 3 counts for 1 mouse. (C) Murine chondrocytes were isolated from rib cartilage of WT or Oprk1 $1^{-/-}$mice. Western blot was performed to detect the expression of lubricin. (D) Chondrocytes were isolated from femoral heads of hip joints of 17-week-old human fetus. Dynorphin A stimulates a dosedependent increase in lubricin expression. (E) Real-time PCR revealed expression of the proteoglycan 4 (PRG4) gene in fetal chondrocytes at the mRNA level. Mean \pm SD $(n=3)$. Each data point represents the average of 3 repeats from 3 tissue donors. (F) Pig articular chondrocytes were treated with KORselective agonist $\mathrm{U} 50,488 \mathrm{H}(1 \mu \mathrm{M})$ for up to 48 hours. Western blot was performed to detect phosphorylation of cAMP responsive element binding protein (CREB) at serine 133. (C) Dynorphin $(1 \mu \mathrm{M})$ treatment increases intracellular concentration of CAMP in chondrocytes. Each data point represents the average of 2 repeats from 3 tissue donors. (H) Fetal chondrocytes were treated with $10 \mu \mathrm{M}$ forskolin (Fsk) for 24 hours. Expression of lubricin was determined by Western blot. (I) PKA inhibitor (KT 5720, $1 \mu \mathrm{M}$ ) reduced lubricin expression and CREB phosphorylation upon dynorphin treatment. (J) Knockdown of PKA catalytic subunit $\alpha$ by siRNA (PRKACA-siRNA) reduced lubricin expression and CREB phosphorylation upon dynorphin treatment. $P$ values in $\mathbf{B}$ and $\mathbf{E}$ were calculated using Student's $t$ test. $P$ values in $\mathbf{G}$ were calculated with 1-way ANOVA followed by Tukey honest significant difference post-hoc test. Blots in panel $\mathbf{C}, \mathbf{D}, \mathbf{F}, \mathbf{H}, \mathbf{I}$, and $\mathbf{J}$ are representative of 1 of $\mathbf{3}$ independent experiments.

similarities. However, triple knockout of 3 major opioid receptors did not show any abnormal phenotype during development (32). Based on these studies, we hypothesized that opioid receptor signaling is not essential for skeletal development, but may have a novel role in cartilage tissue under pathological conditions. Our current study demonstrates that $\mathrm{Oprk1}^{-{ }^{--}}$mice have higher levels of cartilage degeneration after injury and showed an increased expression of catabolic enzymes compared with WT mice. The levels of lubricin were significantly reduced in $O p r k 1^{-1-}$ mice. Subsequent mechanistic studies in vitro showed direct activation of lubricin secretion by KOR agonists.

Previous studies demonstrated that the intracellular mechanisms following KOR activation are cAMP dependent (8), and it was previously reported that KOR activation reduced the intracellular concentration of cAMP in epithelial cells and neurons $(33,34)$. However, other reports also claimed that prolonged exposure to KOR agonist may upregulate adenylyl cyclase and cAMP levels (35). Moreover, dual binding to both inhibitory and excitatory $\mathrm{G}$ proteins has been reported for all opioid receptors (36). Our current data demonstrate that the KOR agonist U50,488H activated the cAMP/CREB signaling pathway in chondrocytes, which is in accordance with the previously published report indicating an increase in cAMP concentration after treatment with dynorphin A in splenic phagocytes (37). 


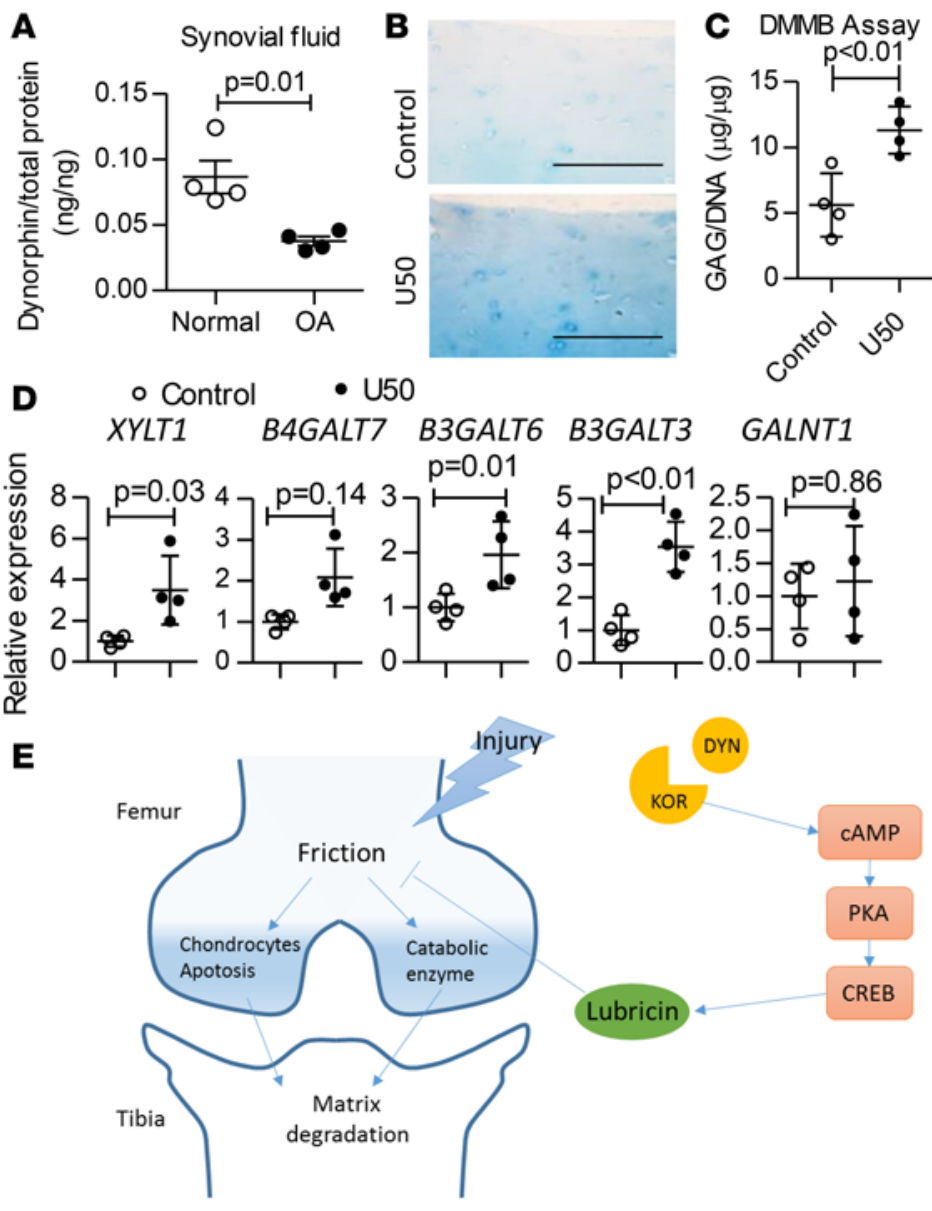

\begin{abstract}
Figure 6. Activation of kappa opioid receptor (KOR) signaling increases glycosaminoglycan content in cartilage explants from osteoarthritis patients. (A) Concentration of dynorphin in synovial fluid of normal individuals and osteoarthritis (OA) patients were measured with ELISA. Mean \pm SD $(n=4)$. Each data point represents an average of 2 repeats for 1 donor. Four normal and $4 \mathrm{OA}$ specimens were used for experimentation. (B) Cartilage explants were obtained from femoral condyles of OA patients who underwent total knee replacement. Explants were treated with $\mathrm{U} 50,488 \mathrm{H}$ (U50) for 1 week. Alcian blue staining was performed to visualize the glycosaminoglycans (GAGs) in cartilage matrix. Scale bars: $100 \mu \mathrm{m}$. Representative images from 4 independent donors are shown. (C) GAG content in explants was measured using 1,9-dimethylmethylene blue (DMMB) assay. Mean \pm SD $(n=4)$. Each data point represents an average of 3 repeats from each of 4 tissue donors. (D) Expression of enzymatic genes that compose the synthetic pathway of chondroitin sulfate. Mean $\pm \operatorname{SD}(n=4)$. $X Y L T 1$, xylosyltransferase I; $\beta 4 G A L T 1, \beta$-1,4-galactosyltransferase, polypeptide 1; $\beta 3$ CALT6, $\beta$-1,3-galactosyltransferase polypeptide 6 ; $\beta 3 C A T 3, \beta-1,3$-glucuronyltransferase 3; GALNT1, UDP- $N$-acetyl- $\alpha$ D-galactosamine:polypeptide $\mathrm{N}$-acetylgalactosaminyltransferase 1. (E) Schematic depicts the model of how KOR/dynorphin (DYN) signaling protects cartilage tissue from posttraumatic degradation. $P$ values were calculated with Student's $t$ test. Each data point represents the average of 3 repeats for 4 tissue donors.
\end{abstract}

It is well established that KOR activation exerts antiinflammatory effects by reducing production of interleukins in many cells types, such as cardiomyocytes (38), monocytes (39), and alveolar osteoclasts (40). KOR expression was also reported in normal synovial fibroblasts and its expression was suppressed under pathological conditions like rheumatic arthritis or OA (41). Moreover, the KOR agonist U50,488H was shown to diminish the development of adjuvant arthritis by reducing cartilage damage (16). Although it is claimed that the KOR agonist was antiinflammatory in these studies, none of these reports had described the molecular mechanisms involved in joint protection after therapy with U50,488H. In this study, we demonstrated that KOR stimulation directly increases lubricin production in articular chondrocytes via cAMP signaling. Additionally, computational predictions indicated that both human and mouse $P R G 4$ had cAMP responsive element (CRE) in the promoter region (42).

Our data also demonstrate that activation of KOR inhibits the expression of catabolic enzymes and cartilage degradation (43). Although the cAMP/CREB signaling pathway was previously shown to play an important role in matrix production by chondrocytes exposed to mechanical loading (44), more studies are needed to dissect the anabolic effects of KOR agonists in cartilage tissue.

Acute full-thickness cartilage injury mimics the response of cartilage and other tissues within the joint to posttraumatic injury. Results presented in the current study show that osteochondral defect in the trochlear groove induced mild or no OA phenotype in femoral condyles of WT mice. Meanwhile, the same defect caused severe cartilage matrix degradation in $\mathrm{Oprk}^{1^{-/-}}$mice.

Addiction is one of the major adverse effects of opioid-like analgesic drugs. It was reported that MOR is the central target of morphine in vivo, and it is a mandatory component of the reward system for morphine dependence (45). Interestingly, KOR agonists do not contribute to the classical drug-seeking behaviors. Unlike MOR agonists, KOR agonists do not cause drug dependence nor do they produce respiratory failure (46); U50,488H actually reduced cocaine and morphine self-administration in rat and mouse models (47). However, KOR activation has also been implicated in producing an array of undesirable side effects, including dysphoric and psychomimetic effects in human clinical studies $(48,49)$. Although these adverse effects limit the therapeutic potential of KOR agonists as analgesics, local intra-articular delivery of these molecules may represent an attractive therapeutic modality for patients with OA.

Taken together, our results show, to our knowledge for the first time, that KOR signaling has direct anabolic and antiapoptotic effects in cartilage cells and also induces lubricin production in chondrocytes via 
the cAMP/CREB pathway. These effects may be beneficial for therapeutic management of patients with degenerative joint disease. Future studies are needed to explore the therapeutic potential of KOR agonists in preclinical models of OA.

\section{Methods}

Isolation of chondrocytes and synovial cells. Normal and OA adult articular cartilage and synovial membrane (2 males and 2 females, 50 to 70 years old, 2-3 on the Kellgren-Lawrence grading scale for OA) were obtained from the National Disease Research Interchange (NDRI). Human fetal aborted tissues were obtained from Novogenix Laboratories following informed consent and elective termination. All donated material was anonymous and carried no personal identity. Using procedures published previously (50), human fetal chondrocytes were obtained from 17-week-old fetal specimens. Yucatan minipig cartilage tissue was obtained from S\&S Farms. Pig chondrocytes were isolated from articular cartilage of Yucatan minipig knee joints. Briefly, the tissue was minced and digested with $1 \mathrm{mg} / \mathrm{ml}$ collagenase II (Worthington) and $1 \mathrm{mg} / \mathrm{ml}$ dispase (Invitrogen) in DMEM/F12 containing 10\% FBS (Sigma-Aldrich) at $37^{\circ} \mathrm{C}$ for more than 16 hours. Pellets were resuspended in PBS and cultured in DMEM/F12 containing 10\% FBS.

siRNA assay. Stealth RNAi, siRNA specific for protein kinase cAMP-activated catalytic subunit $\alpha$ (PRKACA, catalog HSS108468) and subunit $\beta$ (PRKACA, catalog HSS108469), along with a negative control siRNA (catalog 12935300), each containing 19-25 nucleotides, were purchased from Thermo Fisher Scientific. Human chondrocytes were transfected with 25 pmol siRNA and the appropriate amount of transfection reagent Lipofectamine 3000 (Thermo Fisher Scientific) for 72 hours.

FACS. Four mesenchymal subpopulations of cells from digested synovial tissue were isolated by FACS. Briefly, single-cell suspensions were incubated with a mixture of antibodies including APC-conjugated anti-CD31 and anti-CD45 antibodies, PE-conjugated anti-CD34, and PE-Cy7-conjugated anti-CD146 antibodies. Primary antibodies are listed in Supplemental Table 1. All incubations were performed at $37^{\circ} \mathrm{C}$ for 15 minutes in the dark. For sorting, DAPI (diluted 1:1,000; Molecular Probes, catalog D1306) was added to exclude the dead cells. Next, CD31 and CD45 were used to deplete hematopoietic cells and endothelial cells. CD 31-CD45-146+CD34-, CD31-CD45-146-CD34-, CD31-CD45-146-CD34+, and CD31$\mathrm{CD}^{-} 5^{-} 146^{+} \mathrm{CD} 34^{+}$were sorted accordingly. Sorted cells were directly used for RNA extraction.

Annexin $V$ assay. Pig chondrocytes were treated with $10 \mathrm{ng} / \mathrm{ml}$ TNF- $\alpha$ or $10 \mathrm{ng} / \mathrm{ml}$ TNF- $\alpha$ plus $1 \mu \mathrm{M}$ dynorphin A for 48 hours. Next, cells were collected and washed in annexin $\mathrm{V}$ binding buffer and then incubated with PE-conjugated annexin V (BD Biosciences, catalog 556421). Labeled cells were analyzed using a BD LSRII flow cytometer (BD Biosciences). Data files were exported and analyzed with FACSDiva software (BD Biosciences).

Quantitative RT-PCR. Total RNA was isolated from sorted cells or fetal chondrocytes or cartilage explants with the RNeasy Mini Kit (Qiagen). One microgram of total RNA was reverse transcribed into cDNA using the Omniscript RT kit (Qiagen). The primers for quantitative PCR (qPCR) are listed in Supplemental Table 2. qPCR was performed on cDNA samples by using the Maxima SYBR Green/ROX qPCR Master Mix (Thermo Fisher Scientific). PCRs were carried out on a ViiA 7 Real-Time PCR System (Life Technologies). A geometric average of 3 housekeeping genes, B2M, RPL7, and TBP, was used for normalization of relative expression level.

Histology, immunohistochemistry, and immunofluorescence. Mouse or human tissues were fixed in 10\% formalin for 48 hours, dehydrated with ethanol, and embedded in paraffin using routine procedures. A microtome (Leica) was used to cut $5-\mu \mathrm{m}$ sections. Slides were then deparaffinized and stained for sulfated GAG with Alcian blue or safranin O. H\&E staining was performed to assess morphology.

For immunohistochemistry, paraffin sections were processed using routine procedures. Rabbit or mouse antibodies against specific antigens were used, followed by incubation of HRP-conjugated secondary antibodies against rabbit IgG (Vector Laboratories, catalog PI-1000). Primary antibodies are listed in Supplemental Table 1. Antibodies were then visualized by a DAB Peroxidase Substrate Kit (Vector Laboratories).

For immunofluorescence, slides were incubated with primary antibodies, followed by secondary antibody conjugated with AlexaFluor-594 (Invitrogen, catalog A-11037) or AlexaFluor-488 (Invitrogen, catalog A-11008).

Mouse model of cartilage defects in knee joints. C57/BL6 WT and Oprk1-deficient (Oprk1 $\left.1^{-/}\right)$mice were purchased from Jackson Labs. Full-thickness (involving the subchondral bone) cartilage defects were generated in the femoral trochlear groove of a knee joint in mice using a previously described method (20). Briefly, 
4-week-old mice were anesthetized and medial parapatellar arthrotomy was carried out. One-millimeterwide osteochondral defects were made with a Kirshner wire. At weeks 1, 4, and 8, mice were euthanized and dissected for histological examination. All mice used in this study were males. Six WT or Oprk1 ${ }^{-/-}$mice were used for each time point.

OARSI scoring and chondrocyte number counting. The degradation of GAGs in cartilage matrix was assessed after safranin $\mathrm{O}$ staining of sections following OARSI recommendations (21). Three sections from every mouse specimen were scored by 3 blinded researchers. The average numbers from 3 researchers were used to calculate the final score. Chondrocyte numbers were counted on sections after safranin $\mathrm{O}$ staining using ImageJ software (NIH). The area of the cartilage was determined by calibration of a microscope at $\times 100$ magnification.

Cartilage explant cultures. Fetal and adult human cartilage tissue was sliced into small pieces and then cut into uniform tissue pieces by a 1-mm biopsy punch (Miltex, Inc.). Cartilage explants were dispensed in 6-well plates with DMEM supplemented with dynorphin A, U50,488H, or TNF- $\alpha$.

Image acquisition and quantification. All images including bright field and fluorescence were made with an Axio Imager A2 microscope (Zeiss). Quantification of positively stained cells by immunohistochemistry was performed with ImageJ.

$D M M B$ and hydroxyproline assay. Cartilage explants were washed with PBS and frozen overnight at $-80^{\circ} \mathrm{C}$. Subsequently, they were digested with proteinase $\mathrm{K}$ and measured for GAG quantification using spectrophotometrical determination with $\mathrm{DMMB}$ as previously reported (50). Relative cell number was determined by quantification of total DNA using a CyQuant DNA Kit (Molecular Probes, catalog C7026), according to the manufacturer's instructions. For hydroxyproline assay, cartilage explants were digested in papain buffer. The digested solutes were hydrolyzed in $6 \mathrm{~N} \mathrm{HCl}$ at $110^{\circ} \mathrm{C}$ overnight. Hydroxyproline was then assayed spectrophotometrically at $560 \mathrm{~nm}$ after reaction with $0.05 \mathrm{M}$ chloramine- $\mathrm{T}$ and $10 \%$ (w/v in 2-methoxyethanol) $\rho$-dimethylaminobenzaldehyde.

ELISA. Human synovial fluid from healthy and osteoarthritic patients (age 50-70 years old, 2-3 on the Kellgren-Lawrence grading scale for OA) was purchased from Asterand Bioscience. Concentration of dynorphin (combined A and B) in synovial fluid was determined with an ELISA kit (Biomatik) according to the manufacturer's instruction. Normalization was based on total protein concentration in synovial fluid determined by BCA kit (Pierce).

Western blot. After stimulation with dynorphin, U50,488H, or forskolin, chondrocytes were lysed with RIPA buffer with protease and phosphatase inhibitor, at the indicated time points. Lubricin and p-CREB proteins were detected by Western blotting using antibodies as described in Supplemental Table 2. The Western blots were quantified by densitometry using ImageJ software. See complete unedited blots in the supplemental material.

Genotyping. For routine genotyping, genomic DNA was extracted from mouse tails, and PCR was performed (Laragen Inc.).

Statistics. Numbers of repeats for each experiment are indicated in the figure legends. Statistical analysis was performed using 1-way ANOVA followed by Tukey honest significant difference post-hoc test (Prism Software) to compare more than 2 groups, or 2-tailed Student's $t$ test to compare 2 groups. $P$ values less than 0.05 were considered to be significant.

Study approval. All animal studies were performed in accordance with institutional guidelines and approved by the IACUC of the University of Southern California.

\section{Author contributions}

LW and DE designed the research, analyzed the data, and wrote the manuscript. SZ, RS, SL, FG, and CE conducted the experiments. KB and JW analyzed the data. FP analyzed the data and edited the manuscript.YF provided reagents.

\section{Acknowledgments}

The authors are grateful to Felicia Codrea and Jessica Scholes of UCLA for their help with sample analysis and sorting. We would also like to acknowledge Xinmin Li from UCLA CMC for his help with sequencing experiments. This work is supported by NIH grant K01AR061415, Department of Defense grant W81XWH-13-1-0465, and California Institute for Regenerative Medicine grant RB5-07230 (all to DE), as well as National Natural Science Foundation of China (grant 81470721) to YL. 
Address correspondence to: Denis Evseenko, Keck School of Medicine, University of Southern California, 2011 Zonal Avenue, Los Angeles, California 90033, USA. Phone: 1.323.422.2199; E-mail: evseenko@ usc.edu. Or to: Yunfeng Lin, State Key Laboratory of Oral Diseases, West China School of Stomatology, Sichuan University, 14 South Remin Road $3^{\text {rd }}$ section, Chengdu, China, 610041. Phone: 86.28.85503487; E-mail: yunfenglin@scu.edu.cn.

1. Guccione AA, et al. The effects of specific medical conditions on the functional limitations of elders in the Framingham Study. Am J Public Health. 1994;84(3):351-358.

2. Glyn-Jones S, et al. Osteoarthritis. Lancet. 2015;386(9991):376-387.

3. Lawrence RC, et al. Estimates of the prevalence of arthritis and other rheumatic conditions in the United States. Part II. Arthritis Rheum. 2008;58(1):26-35.

4. Johnson VL, Hunter DJ. The epidemiology of osteoarthritis. Best Pract Res Clin Rheumatol. 2014;28(1):5-15.

5. Neogi T, Zhang Y. Epidemiology of osteoarthritis. Rheum Dis Clin North Am. 2013;39(1):1-19.

6. National Clinical Guideline Centre (UK). Osteoarthritis: Care Management in Adults. London, England; National Institute for Health Care Excellence (UK): 2014

7. Spetea M. Opioid receptors and their ligands in the musculoskeletal system and relevance for pain control. Curr Pharm Des. 2013;19(42):7382-7390.

8. Akil H, Watson SJ, Young E, Lewis ME, Khachaturian H, Walker JM. Endogenous opioids: biology and function. Annu Rev Neurosci. 1984;7:223-255.

9. Childers SR. Opioid receptor-coupled second messenger systems. Life Sci. 1991;48(21):1991-2003.

10. Henry DJ, Grandy DK, Lester HA, Davidson N, Chavkin C. Kappa-opioid receptors couple to inwardly rectifying potassium channels when coexpressed by Xenopus oocytes. Mol Pharmacol. 1995;47(3):551-557.

11. Macdonald RL, Werz MA. Dynorphin A decreases voltage-dependent calcium conductance of mouse dorsal root ganglion neurones. J Physiol (Lond). 1986;377:237-249.

12. Bruchas MR, Xu M, Chavkin C. Repeated swim stress induces kappa opioid-mediated activation of extracellular signal-regulated kinase 1/2. Neuroreport. 2008;19(14):1417-1422.

13. Bruchas MR, et al. Long-acting kappa opioid antagonists disrupt receptor signaling and produce noncompetitive effects by activating c-Jun N-terminal kinase. J Biol Chem. 2007;282(41):29803-29811.

14. Rodriguez M, Bard AJ. Electrochemical studies of the interaction of metal chelates with DNA. 4. Voltammetric and electrogenerated chemiluminescent studies of the interaction of tris(2,2'-bipyridine)osmium(II) with DNA. Anal Chem. 1990;62(24):2658-2662.

15. Chavkin C. The therapeutic potential of $\kappa$-opioids for treatment of pain and addiction. Neuropsychopharmacology. 2011;36(1):369-370

16. Bileviciute-Ljungar I, Saxne T, Spetea M. Anti-inflammatory effects of contralateral administration of the kappa-opioid agonist U-50,488H in rats with unilaterally induced adjuvant arthritis. Rheumatology (Oxford). 2006;45(3):295-302.

17. Wu L, et al. Human developmental chondrogenesis as a basis for engineering chondrocytes from pluripotent stem cells. Stem Cell Reports. 2013;1(6):575-589.

18. Crisan M, et al. A perivascular origin for mesenchymal stem cells in multiple human organs. Cell Stem Cell. 2008;3(3):301-313

19. Simonin F, et al. Disruption of the kappa-opioid receptor gene in mice enhances sensitivity to chemical visceral pain, impairs pharmacological actions of the selective kappa-agonist $\mathrm{U}-50,488 \mathrm{H}$ and attenuates morphine withdrawal. EMBO J. 1998;17(4):886-897.

20. Sharifi N, Diehl N, Yaswen L, Brennan MB, Hochgeschwender U. Generation of dynorphin knockout mice. Brain Res Mol Brain Res. 2001;86(1-2):70-75.

21. Regan PM, Langford D, Khalili K. Regulation and functional implications of opioid receptor splicing in opioid pharmacology and HIV pathogenesis. J Cell Physiol. 2016;231(5):976-985.

22. Blasioli DJ, Kaplan DL. The roles of catabolic factors in the development of osteoarthritis. Tissue Eng Part B Rev. 2014;20(4):355-363.

23. Wu L, et al. Extracellular matrix domain formation as an indicator of chondrocyte dedifferentiation and hypertrophy. Tissue Eng Part C Methods. 2014;20(2):160-168.

24. Cuellar A, Reddi AH. Stimulation of superficial zone protein/lubricin/PRG4 by transforming growth factor- $\beta$ in superficial zone articular chondrocytes and modulation by glycosaminoglycans. Tissue Eng Part A. 2015;21(13-14):1973-1981.

25. Iwakura T, Inui A, Reddi AH. Stimulation of superficial zone protein accumulation by hedgehog and Wnt signaling in surface zone bovine articular chondrocytes. Arthritis Rheum. 2013;65(2):408-417.

26. Childers SR, Snyder SH. Guanine nucleotides differentiate agonist and antagonist interactions with opiate receptors. Life Sci. 1978;23(7):759-761.

27. Von Voigtlander PF, Lewis RA. U-50,488, a selective kappa opioid agonist: comparison to other reputed kappa agonists. Prog Neuropsychopharmacol Biol Psychiatry. 1982;6(4-6):467-470.

28. Gonzalez GA, et al. A cluster of phosphorylation sites on the cyclic AMP-regulated nuclear factor CREB predicted by its sequence. Nature. 1989;337(6209):749-752.

29. Sugahara K, Mikami T, Uyama T, Mizuguchi S, Nomura K, Kitagawa H. Recent advances in the structural biology of chondroitin sulfate and dermatan sulfate. Curr Opin Struct Biol. 2003;13(5):612-620.

30. Sora I, et al. Opiate receptor knockout mice define mu receptor roles in endogenous nociceptive responses and morphineinduced analgesia. Proc Natl Acad Sci USA. 1997;94(4):1544-1549.

31. Zhu Y, et al. Retention of supraspinal delta-like analgesia and loss of morphine tolerance in delta opioid receptor knockout mice. Neuron. 1999;24(1):243-252. 
32. Gavériaux-Ruff C, Filliol D, Simonin F, Matthes HW, Kieffer BL. Immunosuppression by delta-opioid antagonist naltrindole: delta- and triple mu/delta/kappa-opioid receptor knockout mice reveal a nonopioid activity. J Pharmacol Exp Ther. 2001;298(3):1193-1198.

33. Yamamizu K, et al. The $\kappa$ opioid system regulates endothelial cell differentiation and pathfinding in vascular development Blood. 2011;118(3):775-785.

34. Kieffer BL, Gavériaux-Ruff C. Exploring the opioid system by gene knockout. Prog Neurobiol. 2002;66(5):285-306.

35. Avidor-Reiss T, Nevo I, Saya D, Bayewitch M, Vogel Z. Opiate-induced adenylyl cyclase superactivation is isozyme-specific. J Biol Chem. 1997;272(8):5040-5047.

36. Crain SM, Shen KF. Opioids can evoke direct receptor-mediated excitatory effects on sensory neurons. Trends Pharmacol Sci. 1990;11(2):77-81

37. Kumar S, Rai U. Dynorphin regulates the phagocytic activity of splenic phagocytes in wall lizards: involvement of a $\kappa-0$ ioid receptor-coupled adenylate-cyclase-cAMP-PKA pathway. J Exp Biol. 2011;214(Pt 24):4217-4222.

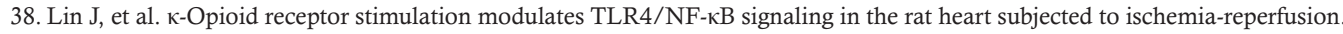
Cytokine. 2013;61(3):842-848.

39. Parkhill AL, Bidlack JM. Reduction of lipopolysaccharide-induced interleukin- 6 production by the kappa opioid U50,488 in a mouse monocyte-like cell line. Int Immunopharmacol. 2006;6(6):1013-1019.

40. Bastos JV, Queiroz-Junior CM, Caliari MV, Francischi JN, Pacheco CM, Maltos KL. Peripheral kappa opioid receptors activation reduces alveolar bone loss in rats by modulating interleukin-6 and -10. Arch Oral Biol. 2011;56(6):540-548.

41. Shen H, et al. Kappa and delta opioid receptors are expressed but down-regulated in fibroblast-like synoviocytes of patients with rheumatoid arthritis and osteoarthritis. Arthritis Rheum. 2005;52(5):1402-1410.

42. Kieffer BL. Recent advances in molecular recognition and signal transduction of active peptides: receptors for opioid peptides Cell Mol Neurobiol. 1995;15(6):615-635.

43. Karsdal MA, et al. Induction of increased cAMP levels in articular chondrocytes blocks matrix metalloproteinase-mediated cartilage degradation, but not aggrecanase-mediated cartilage degradation. Arthritis Rheum. 2007;56(5):1549-1558.

44. Juhász T, et al. Mechanical loading stimulates chondrogenesis via the PKA/CREB-Sox9 and PP2A pathways in chicken micromass cultures. Cell Signal. 2014;26(3):468-482.

45. Matthes HW, et al. Loss of morphine-induced analgesia, reward effect and withdrawal symptoms in mice lacking the muopioid-receptor gene. Nature. 1996;383(6603):819-823.

46. Lalanne L, Ayranci G, Kieffer BL, Lutz PE. The kappa opioid receptor: from addiction to depression, and back. Front Psychiatry. 2014;5:170.

47. Kuzmin AV, Semenova S, Gerrits MA, Zvartau EE, Van Ree JM. Kappa-opioid receptor agonist U50,488H modulates cocaine and morphine self-administration in drug-naive rats and mice. Eur J Pharmacol. 1997;321(3):265-271.

48. Pfeiffer A, Brantl V, Herz A, Emrich HM. Psychotomimesis mediated by kappa opiate receptors. Science. 1986;233(4765):774-776.

49. Roth BL, et al. Salvinorin A: a potent naturally occurring nonnitrogenous kappa opioid selective agonist. Proc Natl Acad Sci USA. 2002;99(18):11934-11939.

50. Wu L, Leijten JC, Georgi N, Post JN, van Blitterswijk CA, Karperien M. Trophic effects of mesenchymal stem cells increase chondrocyte proliferation and matrix formation. Tissue Eng Part A. 2011;17(9-10):1425-1436. 